\title{
Dirac-like monopoles in three dimensions and their possible influences on the dynamics of particles
}

\author{
E. M. C. Abreu* and M. Hott ${ }^{\dagger}$ \\ Departamento de Física e Química, UNESP/Guaratinguetá, Caixa Postal 205, Guaratinguetá, \\ CEP 12516-410, São Paulo, Brazil \\ J. A. Helayël-Neto \\ Centro Brasileiro de Pesquisas Físicas, Rua Xavier Sigaud 150, Urca, CEP 22290-180, Rio de Janeiro, \\ Rio de Janeiro, Brazil \\ and Grupo de Física Teórica, Universidade Católica de Petrópolis, Rua Barão do Amazonas 124, \\ CEP 25685-070, Petrópolis, Rio de Janeiro, Brazil \\ Winder A. Moura-Melo ${ }^{\S}$ \\ Departamento de Ciências Exatas, Universidade Federal de Lavras, Caixa Postal 37, CEP 37200-000, Lavras, Minas Gerais, Brazil
}

(Received 19 December 2001; published 5 April 2002)

\begin{abstract}
Dirac-like monopoles are studied in three-dimensional Abelian Maxwell and Maxwell-Chern-Simons models. Their scalar nature is highlighted and discussed through a dimensional reduction of four-dimensional electrodynamics with electric and magnetic sources. Some general properties and similarities whether considered in Minkowski or Euclidean space are mentioned. However, by virtue of the structure of the space-time in which they are studied, a number of differences among them occur. Furthermore, we pay attention to some consequences of these objects when they act upon the usual particles. Among other subjects, special attention is given to the study of a Lorentz-violating nonminimal coupling between neutral fermions and the field generated by a monopole alone. In addition, an analogue of the Aharonov-Casher effect is discussed in this framework.
\end{abstract}

DOI: $10.1103 /$ PhysRevD.65.085024

PACS number(s): 11.10.Kk, 11.15.-q

\section{INTRODUCTION AND MOTIVATION}

The idea that magnetic monopoles, as stable particles carrying magnetic charges, ought to exist has proved to be remarkably durable. In $3+1$ dimensions, a persuasive argument was first put forward by Dirac in 1931 [1], who invoked such objects in order to provide a theoretical explanation of why electric charges appear only as multiples of an elementary one.

Furthermore, 't Hooft [2] and Polyakov [3] discovered that the existence of magnetic monopoles follows from quite general ideas about the unification of the fundamental interactions. Nowadays, it is well known that such objects emerge from general "grand unified" theories of particle physics whose gauge group is suitably broken down to the U(1) factor. Indeed, Dirac proved the consistency of structureless magnetic monopoles with quantum electrodynamics. On the other hand, some properties of the 't Hooft-Polyakov monopole, such as its size and mass, are determined by the distance scale of the spontaneous symmetry breakdown of a grand unified theory. The magnetic charge $g$ of the monopole is typically the "Dirac charge," $g_{D}=1 / 2 e$, which is distributed over a core with a radius of order $M_{X}^{-1}$ (the unification

\footnotetext{
*Electronic address: everton@feg.unesp.br

†Electronic address: hott@feg.unesp.br

‡Electronic address: helayel@cbpf.br

${ }^{\S}$ Electronic address: winder@stout.ufla.br
}

distance scale) while its mass is comparable to the magnetostatic potential energy of the core. An excellent review of these subjects may be found in Ref. [4].

In turn, the study of three-dimensional field theories has attracted a great deal of interest for nearly two decades $[5,6]$. Even though such studies were initially motivated by the theoretical connection between such models and their fourdimensional analogues at high temperature, planar physics enjoys nowadays the status of an interesting and selfcontained topic in itself. This position was achieved, in part, thanks to some peculiar features that occur in this spacetime, such as the coexistence of massive vector gauge bosons and gauge invariance, and the possibility of having objects displaying charge and statistical fractionization $[7,8]$. On the other hand, the interest in planar physical models was also remarkably motivated by condensed matter phenomena that display planar dynamics. Among these, we may quote the quantum Hall effect [9] and high- $T_{c}$ superconductivity $[8,10]$.

Of particular interest is also the study of topological objects in this framework. For example, topologically magnetic vortexlike solutions naturally appear attached to electric charges whenever we are dealing with a Chern-Simons-like electrodynamics [the so-called Maxwell-Chern-Simons (MCS) model]. In addition, it is well known that this composite entity (electric charge + magnetic vortex) may present anyonic statistics because of the magnetic flux induced by the vortex $[8,11]$.

Another sort of topological entity shows up whenever the Bianchi identity is broken. These are generally characterized by a potential $A_{\mu}$ that carries a singular structure. As is well 
known, such potentials first appeared in Dirac's paper on magnetic monopoles [1]. Actually, while in $3+1$ dimensions the simplest solution appears as a pointlike magnetic monopole, we shall see that in the $(2+1)$-dimensional case the breaking of the Bianchi identity leads us to a wider class of solutions, not restricted to magnetic ones (this is the reason that we call them Dirac-like objects).

Indeed, some work has dealt with such issues in both Euclidean [12,13] and Minkowskian [14] three-dimensional spaces. Here, it is worth mentioning that the mass parameter was shown to be quantized in the Abelian version of the Maxwell-Chern-Simons model whenever Dirac-like monopoles interact with the usual charges [12] (similar to the result already known for theories whose gauge groups present the nontrivial third homotopy group [6]). In addition, classical and quantum consequences of the monopole potential acting upon a charged particle were recently analyzed [14].

In this article we wish to go further into this subject and investigate some issues concerning the nature of such objects in three dimensions, as well as some of their influences on the dynamics of particles. Thus, in Sec. II we introduce a dimensional reduction of $(3+1) \mathrm{D}$ electrodynamics with magnetic sources to $2+1$ dimensions. This presentation is interesting for highlighting the scalar nature of these sources in the planar case. Indeed, such a scheme yields two Abelian "electrodynamics" which do not have any explicit interplay between them. In addition, we point out the differences between these models, particularly in their magnetic sectors.

Section III is devoted to the subject of the Dirac-like monopole itself. There, we present a brief review of such objects introduced in Minkowskian and Euclidean spaces. Attention is given to the differences between them. We also present an analysis of the solutions admitted by the differential equation that shows up whenever the Bianchi identity is broken in $2+1$ dimensions.

In Sec. IV, we deal with the interaction between a Diraclike monopole and the usual particle. More precisely, our attention is focused on a Lorentz-violating nonminimal term, which couples monopole field strength to neutral matter. Although violating Lorentz, it is shown to be invariant under $C P T$ symmetry. In addition, the equations of motion are similar to those we have for the case of a charged particle minimally interacting with the vector potential produced by a magnetic vortex. Indeed, by virtue of this similarity, this interaction leads us to an Aharonov-Casher-like effect on the usual particle, produced by the tangential electric field of the monopole.

Finally, our paper is closed by pointing out our conclusions and prospects for future investigation.

\section{THE ORIGIN OF THE SCALAR NATURE OF PLANAR DIRAC-LIKE OBJECTS}

Here, we intend to give an alternative view of the scalar nature of Dirac-like monopoles in $2+1$ dimensions. The proper study of the breaking of the Bianchi identity in planar Abelian Maxwell and Maxwell-Chern-Simons frameworks will be the goal of the next section, where we shall pay attention, among other things, to the tangential (azimuthal) behavior of the electriclike field generated by a pointlike "magnetic source" [13,14].

In order to trace back the scalar nature of a $(2+1) \mathrm{D}$ magnetic current to its four-dimensional ancestor, we propose to carry out a plan dimensional reduction of the $(3+1)$ D Maxwell theory with electric $\left(j^{\hat{\mu}}\right)$ and magnetic $\left(k^{\hat{\mu}}\right)$ sources, Eqs. (1),(2) below, to the planar case. Hereafter, we shall work in Minkowski space-time, but no difficulty arises in carrying out a similar plan in the Euclidean case.

We start off from ${ }^{1}$

$$
\begin{aligned}
& \partial_{\hat{\mu}} F^{\hat{\mu} \hat{\nu}}=j^{\hat{\nu}}, \\
& \partial_{\hat{\mu}} \widetilde{F}^{\hat{\mu} \hat{\nu}}=k^{\hat{\nu}},
\end{aligned}
$$

with $F^{\hat{\mu} \hat{\nu}}=\partial^{\hat{\mu}} A^{\hat{\nu}}-\partial^{\hat{\nu}} A^{\hat{\mu}}$ and $\widetilde{F}^{\hat{\mu} \hat{\nu}}=\frac{1}{2} \epsilon^{\hat{\mu} \hat{\nu} \hat{\kappa} \hat{\lambda}} F_{\hat{\kappa} \hat{\lambda}}$.

First of all, we reduce the potential and currents as in the "splitting" below:

$$
\begin{gathered}
A^{\hat{\mu}} \rightarrow\left(A^{\mu} ; A^{3} \equiv S\right), \\
j^{\hat{\mu}} \rightarrow\left(j^{\mu} ; j^{3} \equiv \lambda\right), \\
k^{\hat{\mu}} \rightarrow\left(k^{\mu} ; k^{3} \equiv \chi\right) .
\end{gathered}
$$

Then, we realize that the $(3+1)$-dimensional quantities are reduced to $(2+1) \mathrm{D}$ ones. For instance, $A^{\hat{\mu}}$ yields a $(2+1) \mathrm{D}$ vector $A^{\mu}=\left(A^{0}, A^{1}, A^{2}\right)$, and an extra scalar potential $A^{3}$ $\equiv S$. Notice, in addition, that from the point of view of a $(2+1)$-dimensional frame the fields $A^{\mu}$ and $S$ are, in principle, completely independent (the same is valid for the currents). Similarly, $j^{\mu}$ and $k^{\mu}$ are the $(2+1) \mathrm{D}$ electric and magnetic currents, while $j^{3} \equiv \lambda$ and $k^{3} \equiv \chi$ represent the survivors of the third components of the electric and magnetic genuine four-currents, respectively.

In addition, adopting the reduction ansatz that the quantities do not depend on the third spatial coordinate, say, $\partial_{3}(f)=0$, where $f$ represents any potential or current, the field strengths take the following forms after the dimensional reduction:

$$
\begin{aligned}
& F^{\hat{\mu} \hat{\nu}} \rightarrow\left(F^{\mu \nu} ; F^{\mu 3} \equiv G^{\mu}\right), \\
& \widetilde{F}^{\hat{\mu} \hat{\nu}} \rightarrow\left(\widetilde{F}^{\mu \nu} \equiv \widetilde{G}^{\mu \nu} ; \widetilde{F}^{\mu 3}=\widetilde{F}^{\mu}\right),
\end{aligned}
$$

where the new field strengths are defined as $F^{\mu \nu}=\partial^{\mu} A^{\nu}$ $-\partial^{\nu} A^{\mu}, \widetilde{F}^{\mu}=\frac{1}{2} \epsilon^{\mu \nu \kappa} F_{\nu \kappa}, G^{\mu}=\partial^{\mu} S$, and $\widetilde{G}^{\mu \nu}=\epsilon^{\mu \nu \kappa} G_{\kappa}$.

Notice also that the usual planar electric and magnetic fields are contained in the former field, say, $\widetilde{F}^{\mu}=(-B$; $\left.-\epsilon^{i j} E^{j}\right)$. In turn, the fields $G^{\mu}=\partial^{\mu} S$ and $\widetilde{G}^{\mu \nu}=\epsilon^{\mu \nu \kappa} G_{\kappa}$ indicate the appearance of another "electrodynamic" model, as shown below.

\footnotetext{
${ }^{1}$ Our conventions read $\hat{\mu}, \hat{\nu}$, etc. $=0,1,2,3$, diag $\eta_{\hat{\mu} \hat{\nu}}=(+,-,-$, $-)$, and $\epsilon^{0123}=-\epsilon_{0123}=+1$. In addition, $\mu, \nu$, etc. $=0,1,2$, $\operatorname{diag} \eta_{\mu \nu}=(+,-,-)$, and $\epsilon^{012}=\epsilon_{012}=+1$; while the planar spatial indices are labeled as $i, j=1,2$ and $\epsilon^{12}=\epsilon_{12}=+1$.
} 
Now, taking into account relations (4)-(7), expressions (1),(2) lead us to the two following sets of equations:

$$
\begin{array}{ccc}
\partial_{\mu} F^{\mu \nu}=j^{\nu} & \text { and } & \partial_{\mu} \widetilde{F}^{\mu}=\chi, \\
\partial_{\mu} G^{\mu}=\lambda & \text { and } & \partial_{\mu} \widetilde{G}^{\mu \nu}=k^{\nu},
\end{array}
$$

from which we may still write down

$$
\begin{aligned}
\epsilon^{i j} \partial^{i} B & =\partial_{t} E^{j}+j^{j}, \\
\nabla \cdot \vec{E}=j^{0} & =\rho, \\
\partial_{t} B+\epsilon^{i j} \partial^{i} E^{j} & =\chi, \\
\epsilon^{i j} \partial^{i} b & =\epsilon^{i j} \partial_{t} e^{i}+k^{j}, \\
\epsilon^{i j} \partial^{i} e^{j} & =k^{0}=\rho_{m}, \\
\partial_{t} b-\nabla \cdot \vec{e} & =\lambda,
\end{aligned}
$$

where the fields above are defined as

$$
\begin{aligned}
& E^{i}=-\partial^{i} A^{0}-\partial_{t} A^{i} \quad \text { and } \quad B=\epsilon^{i j} \partial^{i} A^{j}, \\
& e^{i}=-\partial^{i} S \quad \text { and } \quad b=\partial_{t} S .
\end{aligned}
$$

Therefore, we realize that after dimensional reduction is implemented we get two independent electrodynamic like models in $2+1$ dimensions, each of them with its proper electric and magnetic sources. Indeed, the appearance of two noncoupled Abelian factors is nothing but a natural consequence of the reduction scheme. For instance, the latter is equivalent to selecting the zero-mode sector of a more general dimensional reduction proposal, namely, the KaluzaKlein ansatz that relies on the compactness of the third spatial coordinate [15]. Thus, the natural $\mathrm{SO}(2)$ symmetry associated with such a component is kept in $(2+1) \mathrm{D}$, since the scalar field $S$ is clearly invariant under rotations in the plane. We should also notice that the number of on-shell degrees of freedom is conserved in the reduction scheme. The two physical components of $A^{\hat{\mu}}$ lie, after dimensional reduction, in $A^{\mu}$ and in $S$, each of them carrying a unique degree of freedom.

Furthermore, it is important to stress here that the breaking down of the Bianchi identity in $(2+1) \mathrm{D}$ and what we interpret as its associated magnetic source in the planar world is the $(2+1) \mathrm{D}$ manifestation of the third component of the genuine magnetic four-current. ${ }^{2}$ This is how we understand the argument by Henneaux and Teitelboim [12] that this charge rather behaves like an instanton in the planar

\footnotetext{
${ }^{2}$ For this, notice that we are considering, as is usually done, the set of equations (8) as being the (2+1)-dimensional counterpart of the standard electrodynamics in four dimensions. The other Abelian sector, Eq. (9), that comes from the scalar potential $S$ is then merely considered as being the partner of planar electromagnetism after the reduction procedure, even though the set (9) is the one that keeps the "genuine" $(2+1) \mathrm{D}$ reminiscent of the magnetic four-current.
}

case. In addition, it is worth noticing that the $\chi$ charge is a pseudoscalar; it changes its signal under parity, $\chi \rightarrow \chi^{P}=$ $-\chi$, which is consistent with the equations of motion and with the fact that it appears, after dimensional reduction, as a reminder of the magnetic four-current, which is a pseudovector. Similar behavior also occurs for all other currents and fields above. It would also be interesting to understand now, if it is possible to accomplish such a program, how the (3 +1 )-dimensional Dirac quantization condition may induce an analogue in the $\chi$ charge.

So our claim is that, once we start with a 4D Maxwell theory enriched by the presence of magnetic monopoles, and if some physical system is considered such that nonplanar effects are negligible in comparison with planar effects, the system may reveal particles that interact via two quantum numbers and one of them may induce an electric field with azimuthal configuration (see Sec. III for details).

\section{ANALYZING THE BREAKING OF THE BIANCHI IDENTITY}

Dirac-like objects come about through breaking the Bianchi identity, as we have already mentioned. In $(3+1)$ dimensions, when we consider Maxwell electrodynamics with magnetic sources, we have the equations $\partial_{\hat{\mu}} F^{\hat{\mu} \hat{\nu}}=j^{\hat{\nu}}$ and $\partial_{\hat{\mu}} \widetilde{F}^{\hat{\mu} \hat{\nu}}=k^{\hat{\nu}}$. There, the magnetic Gauss law $\nabla \cdot \vec{B}=\chi^{0}$, whenever taken for a pointlike source $\chi^{0}=g \delta^{3}(\vec{x})$, leads us to the concept of a genuine magnetic monopole since $\vec{B}$ $=g \vec{x} / 4 \pi|\vec{x}|^{3}$, in analogy with the electric field produced by an isolated pointlike electric charge. Clearly, this similarity occurs because of the duality between electric and magnetic sectors, i.e., $\vec{E}$ and $\vec{B}$ are rank-1 tensors (notice that this happens only in four dimensions).

On the other hand, when considered in $2+1$ dimensions, the broken version of the Bianchi identity yields

$$
\partial^{\mu} \widetilde{F}_{\mu}=\partial_{t} B+\epsilon^{i j} \partial^{i} E^{j}=\chi .
$$

Here, there is no Gauss law for the magnetic field, which implies, in turn, that magnetic monopoles like those we encountered in four dimensions are no longer present. Thus, although arising like genuine magnetic sources in $(3+1)$-dimensional electromagnetism, the present objects are expected to exhibit several differences when compared to the first ones.

Furthermore, in dealing with the massless case the breaking of the Bianchi identity causes no effect on the equations of motion, i.e., electric current is automatically conserved,

$$
\partial_{\nu} \partial_{\mu} F^{\mu \nu}=\partial_{\nu} j^{\nu}=0 .
$$

Nevertheless, when the Chern-Simons term $\mathcal{L}_{C S}=m A_{\mu} \widetilde{F}^{\mu}$ is taken into account, things change greatly. Now, the equations of motion acquire an extra (topological) current term,

$$
\partial_{\mu} F^{\mu \nu}=j^{\nu}+m \widetilde{F}^{\nu},
$$

which yields $\nabla \cdot \vec{E}=\rho+m B$ and $\epsilon^{i j} \partial^{i} B=\partial_{t} E^{j}+j^{j}+m \epsilon^{i j} E^{i}$. 
Now, contrary to the massless case, if Dirac-like objects are introduced, $\partial^{\mu} \widetilde{F}_{\mu}=\chi$, then current is no longer conserved, i.e.,

$$
\partial_{\nu} \partial_{\mu} F^{\mu \nu}=m \chi,
$$

and gauge symmetry is lost. Thus, in order to restore such symmetry we should suppose that the appearance of Diraclike entities naturally induces an extra electric current

$$
j_{M}^{\nu}=-m \widetilde{F}^{\nu},
$$

so that Eq. (12) is modified to

$$
\partial_{\mu} F^{\mu \nu}=J^{\nu}+m \widetilde{F}^{\nu},
$$

and it is now identically conserved,

$$
\partial_{\nu} \partial_{\mu} F^{\mu \nu}=\partial_{\nu} J^{\nu}+m \partial_{\nu} \widetilde{F}^{\nu}=0,
$$

where $J^{\nu}=j^{\nu}+j_{M}^{\nu}$ is the total (usual+topologically induced) electric current (for further details, see Refs. [12,13]).

On the other hand, in the three-dimensional Euclidean space, we have that

$$
\partial_{\mu} \widetilde{F}^{\mu}=\partial_{\tau} \widetilde{F}^{0}+\partial_{i} \widetilde{F}^{i}=\chi .
$$

Now, writing $\widetilde{F}^{\mu}=-\partial^{\mu} \phi$ and taking $\chi$ as being a point, $\chi$ $=g \delta^{3}(\vec{x})$, we get $\partial^{2} \phi=-g \delta^{3}(\vec{x})$, whose solution reads

$$
\phi(\vec{x})=-g / 4 \pi|\vec{x}|,
$$

where $|\vec{x}|=\sqrt{\tau^{2}+x_{i}^{2}}$.

The fields, in turn, are given by $\widetilde{F}^{\mu}=-\partial^{\mu} \phi$ $=g x^{\mu} / 4 \pi|\vec{x}|^{3}$, or [let us recall that $\left.\widetilde{F}^{\mu}=\left(-B ;-\epsilon^{i j} E^{j}\right)\right]$ :

$$
B=-\frac{g}{4 \pi} \frac{x^{0}}{|\vec{x}|^{3}} \quad \text { and } \quad E_{i}=-\frac{g}{4 \pi} \frac{\epsilon_{i j} x_{j}}{|\vec{x}|^{3}},
$$

which clearly shows us that genuine magnetic monopoles, whose only effect is the production of a magnetic field, such as we realized in four dimensions, no longer occurs here.

The tangential character of the electric field above is particularly noticeable (like its analogue in the Minkowski case, below), in contrast to what we expect from the usual electric or even magnetic poles. When working in the MCS framework, the induced electric current, Eq. (14), is readily found to be

$$
\rho_{M}=-\frac{m g}{4 \pi} \frac{\tau}{|\vec{x}|^{3}} \quad \text { and } \quad j_{M}^{i}=-\frac{m g}{4 \pi} \frac{x^{i}}{|\vec{x}|^{3}}
$$

which presents a radial-like dependence. Further details about this subject, including investigation of non-Abelian versions of such entities, may be found in Ref. [13].

Now, let us return to Minkowski space-time and let us analyze the structure and solutions of Eq. (10) in detail. Rewriting this equation in components we get (hereafter, we shall use a subscript $g$ in order to distinguish these fields from the usual electric and magnetic ones and from their Euclidean counterparts),

$$
\partial_{t} B_{g}+\epsilon^{i j} \partial^{i} E_{g}^{j}=\chi,
$$

whose pointlike solutions may be obtained by considering special situations. First, considering the static limit of the fields in the equation above, we obtain

$$
\epsilon^{i j} \partial^{i} E_{g}^{j}=g \delta^{2}(x),
$$

which, when written in terms of the potential $\vec{E}_{g}=-\nabla \Phi_{g}$, has the following form:

$$
\left[\partial_{x}, \partial_{y}\right] \Phi_{g}=-g \delta^{2}(\vec{x}),
$$

whose solution reads [with $r=|\vec{r}|=\sqrt{x^{2}+y^{2}}$ and $\theta$ $=\arctan (y / x)$, as usual]

$$
\Phi_{g}(\vec{x})=-\frac{g}{2 \pi} \arctan \left(\frac{y}{x}\right)=-\frac{g}{2 \pi} \theta .
$$

Notice the remarkable feature of this potential: it has angular rather than radial dependence. Notice also its singular structure: the angle function is not well defined at the origin, like the stringlike structure presented by the vector potential associated with a genuine magnetic monopole in $3+1$ dimensions. In addition, it is a multivalued function and the corresponding electric field (see below) is not a conservative one, a fact already indicated by Eq. (22). Indeed, its associated electric field reads

$$
\vec{E}_{g}=\frac{g}{2 \pi} \frac{x \hat{j}-y \hat{i}}{x^{2}+y^{2}}=\frac{g}{2 \pi} \frac{\hat{e}_{\theta}}{r}
$$

as in Ref. [14], which has an azimuthal rather than a radiallike vector behavior. In addition, by demanding null radiation at this static limit, $\int_{V} \partial_{i} \epsilon_{i j} E_{j} B d V=0$, it readily follows that in this case $B_{g}$ must vanish. Therefore this static solution appears to be due to a peculiar topological electric charge, rather than to a magnetic monopole.

Furthermore, if we compare it with the vector potential associated with a magnetic vortex $\left(\Phi_{B}\right.$ being its magnetic flux),

$$
\vec{A}_{v}(\vec{x})=\frac{\Phi_{B}}{2 \pi r} \hat{e}_{\theta},
$$

we may identify a kind of "duality" between them. Actually, the magnetic vortex may be obtained from a Dirac-like monopole, Eq. (25), by interchanging the vectors $\vec{A}_{v}$ and $\vec{E}_{g}$, together with $g$ and $\Phi_{B}$ (let us recall that in the case of the usual electric charge a similar identification requires the interchanging of $\vec{A}_{v}$ and the dual of $\vec{E}$ ).

Before carrying on the analysis of other possible solutions, let us pay attention to the (topological) electric current induced by the appearance of this monopole in the MCS framework. From Eqs. (14) and (25) it follows that 


$$
\rho_{M}=0 \quad \text { and } \quad j_{M}^{i}=\frac{m g}{2 \pi} \frac{x^{i}}{|\vec{x}|^{2}},
$$

which is radial, and implies that Eq. (16) is satisfied.

The second situation is the radial-like electric field. Now, searching for solutions of Eq. (21) that present $\epsilon^{i j} \partial^{i} E^{j}=0$, we are left with

$$
\partial_{t} B_{g}=\chi
$$

Here, let us take the simplest time-dependent configuration for the $\chi$ charge, $\chi=g \delta(t) \delta^{2}(\vec{x})$, which is similar to the one we have taken in Euclidean space. This case is readily solved by taking

$$
B_{g}(\vec{x}, t)=g \delta^{2}(\vec{x}) \Theta(t),
$$

which is clearly the magnetic field due to a vortexlike object with flux equal to $2 \pi g$ (created at $t=0)$.

We may also think about a configuration that reverses the direction of the magnetic flux, say,

$$
B_{g}(\vec{x}, t)=(g / 2) \delta^{2}(\vec{x})[\Theta(t)-\Theta(-t)],
$$

which clearly represents a magnetic vortex with flux $-g / 2$ that changes its signal at $t=0$, or the destruction of a $-g / 2$ flux vortex at $t=0$ with the simultaneous creation of another one with flux $g / 2$.

For such an object Eq. (29), its topologically induced electric current, takes the form

$$
\rho_{M}=m g \delta^{2}(\vec{x}) \Theta(t) \quad \text { and } \quad j_{M}^{i}=0
$$

which represents a pointlike electric charge of strength $-m g$ created at $t=0$. In addition, since $B$ and $\rho_{M}$ above are located at $\vec{x}=0$, we conclude that in the MCS case the appearance of a composite vortex-electric charge may be alternatively provided through the introduction of a vortexlike solution, such as Eq. (29), whenever the Bianchi identity is broken.

A more general solution associated with Eq. (21) is obtained by taking a "mixture" of previous ones. Let $\chi$ $=g \delta^{2}(\vec{x}) \delta(t)$ and let us combine previous solutions, as shown:

$$
B_{g}(\vec{x}, t)=\frac{g}{2} \delta^{2}(\vec{x}) \Theta(t) \quad \text { and } \quad \vec{E}_{g}=+\frac{g}{4 \pi} \frac{\hat{e}_{\theta}}{|\vec{x}|} \delta(t)
$$

As is clear, such expressions bring together the solutions associated with the vortexlike solution, created at $t=0$, and the Dirac-like monopole, only at $t=0$. The electric field above induces

$$
\vec{j}_{M}=\frac{m g}{4 \pi} \frac{\vec{x}}{|\vec{x}|^{2}} \delta(t)
$$

which takes electric charges away from the origin at $t=0$, while

$$
\rho_{M}=\frac{m g}{2} \delta^{2}(\vec{x}) \Theta(t)
$$

corresponds to the induced charge at $\vec{x}=0$, provided by $\vec{j}_{M}$.

Let us compare the solution above with that we have in Euclidean space, Eq. (19). The monopolelike solution in Euclidean space, Eq. (19), represents an object that produces a tangential electric field and a "radial-like" magnetic field, both of them proportional to $1 /|\vec{x}|^{2}$. On the other hand, if we consider one of its analogues in Minkowskian space-time, solution (32), we realize that in this case the monopolelike solution gives us a magnetic field confined to a point in space, a vortex, and a tangentially directed electric field which is proportional to $1 /|\vec{x}|$ and, in addition, occurs only at $t=0$. Therefore, we conclude that the dimension and structure (topology, etc.) of the space-time are decisive for the solutions of the fields associated with Dirac-like objects.

Before closing this section, let us pay attention to the issue concerning the introduction of such entities in electrodynamiclike models, namely, three-dimensional Abelian gauge theories. First of all, notice that in the Bianchi identity breaking scenario, no space is reserved for the appearance of a mass term, i.e., we could not provide a mass gap for the radiation associated with the monopolelike field (for the time being, we are supposing different radiation for the dynamical and geometrical sectors of the equations of motion).

Now, in the case of the pure Maxwell (massless) model, the breaking of the Bianchi identity causes no additional trouble in the dynamical sector; for instance, electric current remains conserved. Therefore, in this case, nothing prevents us from taking into account that we have indeed a unique (massless) radiation which mediates the interaction among the usual electric charges (the usual electric and magnetic fields), among Dirac-like objects $\left(\vec{E}_{g}\right.$ and $\left.B_{g}\right)$, and also among both kinds of particles. It is worth noticing that such an identification of apparently distinct sorts of interaction as being manifestations of only one kind of radiation is possible here because all the required potentials and fields are gapless.

However, if we try to apply a similar identification in the MCS framework we meet serious trouble. Here, the usual radiation is naturally massive. For example, the electric field between two static electric charges is proportional to $K_{0}\left(m|\vec{x}|\right.$ ) (with $K_{0}$ being the modified Bessel function of the second kind at zeroth order), and so it is a short-range interaction. In great contrast, the tangential electric field due to a monopolelike solution carries no hint about mass; see Eqs. (19) and (25). Actually, as far as we have tried, no way was found to identify both types of interaction as produced by the same radiation. This would require an action which has already enclosed the usual and monopolelike potentials as its basic ingredients, and so, would answer whether one or two kinds of radiation are required.

\section{NEUTRAL PARTICLES NONMINIMALLY COUPLED TO MONOPOLE FIELD AND THE AHARONOV-CASHER EFFECT}

In this section we shall consider a nonminimal coupling of a spinor field with the electric field generated by a "static 
monopole," Eq. (25). First, however, we briefly review some basic aspects of the usual nonminimal case, mainly those concerning the Aharonov-Casher effect. Indeed, it is a peculiarity of $2+1$ dimensions that even spinless particles may carry anomalous magnetic momentum, whenever they interact with an electromagnetic field. The reason lies in the fact that the momentum may be naturally supplemented by a dual field strength, say,

$$
\partial_{\mu} \rightarrow \partial_{\mu}+i h \widetilde{F}_{\mu}
$$

where $h$ measures the planar anomalous magnetic momentum of the matter (see, for example, Refs. [16,17], for further details).

Now, let us take the electromagnetic field $\widetilde{F}_{\mu}=$ $\left(-B ; \epsilon_{i j} E_{j}\right)$ produced by the usual pointlike electric charge, say, $B=0$ and $\vec{E}=q \vec{x} / 2 \pi|\vec{x}|^{2}$. Then, if we consider the interaction of such a field with a given particle (mass $m$ ), we find that the energy operator of the latter reads

$$
H=\frac{1}{2 m}\left(\partial_{i}+i h \widetilde{F}_{i}\right)^{2}=\frac{1}{2 m}\left(\partial_{i}+i h \epsilon_{i j} E_{j}\right)^{2} .
$$

In addition, if the "free" wave functions associated with the particle satisfy

$$
\left(i \partial_{0}+\frac{1}{2 m} \nabla^{2}\right) \psi^{(0)}=0
$$

then the WKB approximation yields the new functions

$$
\psi(\vec{x}, t)=\psi^{(0)}(\vec{x}, t) \exp \left[-i \int d x_{\mu} h \widetilde{F}^{\mu}\right]
$$

Thus we realize that the addition of the field $\widetilde{F}^{\mu}$ to the usual momentum is equivalent to introducing (at the WKB level) a nonintegrable phase to the former wave functions. Clearly, a similar plan also holds in the case of the minimal coupling $\partial_{\mu} \rightarrow \partial_{\mu}+i e A_{\mu}$ (see Ref. [17]), which is responsible for the appearance of the celebrated Aharonov-Bohm (AB) effect [18] and in 2+ 1 dimensions magnetic flux-carrying particles lead to fractional statistics [8].

The interesting point to be noticed here is that, if we consider that the particle performs a spatial loop, say $\theta$, around the charge $q$, then

$$
\theta=h \oint d l_{i} \widetilde{F}^{i}=h \oint d l_{i} \epsilon^{i j} E^{j}=\frac{h q}{2 \pi} \oint d l_{i} \epsilon^{i j} \frac{x^{j}}{|\vec{x}|^{2}}
$$

Now, since $d l_{i}=\epsilon_{i j} d x_{j} \quad(d \vec{x}$ is radial $)$ and $\nabla \cdot \vec{x} /|\vec{x}|^{2}$ $=2 \pi \delta^{2}(\vec{x})$, we finally obtain

$$
\theta=\frac{h q}{2 \pi} \int_{S} d S \nabla \cdot \frac{\vec{x}}{|\vec{x}|^{2}}=h q
$$

Therefore, we have that $\psi(\vec{x}, t)=\psi^{(0)}(\vec{x}, t) e^{i \theta}$, where $\theta$ is the Aharonov-Casher (AC) phase provided by the electric field $\vec{E}=q \vec{x} / 2 \pi|\vec{x}|^{2}$ (for further details, see Refs. [17,19,21], and related references therein).

In our present case, the counterpart of the electric field above reads like Eq. (25), $\vec{E}_{g}=g(x \hat{j}-y \hat{i}) / 2 \pi|\vec{x}|^{2}$. Then, $\widetilde{F}_{g}^{i}=-\epsilon^{i j} E_{g}^{j}=g \vec{x} / 2 \pi|\vec{x}|^{2}$ is already radial. In this case, a similar loop as in the previous case, $\theta^{\prime}$, vanishes:

$$
\theta^{\prime}=h \oint d l_{i} \widetilde{F}^{i}=\frac{h g}{2 \pi} \oint d l_{i} \frac{x^{i}}{|\vec{x}|^{2}}=0
$$

Thus our monopole does not induce an AC phase on a given particle if they interact in the usual nonminimal way. In addition, we should notice that the contrast between the cases above comes from the fact that, in the first one, i.e., $\vec{E}$ $=q \vec{x} / 2 \pi|\vec{x}|^{2}$, the dual operation induced whenever taking $\widetilde{F}^{i}=-\epsilon^{i j} E^{j}$ is exactly compensated by an extra term associated with $d l_{i}=\epsilon_{i j} d x_{j}$.

In view of this aspect, we shall consider here the (Lorentz-odd) nonminimal terms like the one below (coupled, for concreteness, to spinors):

$$
\mathcal{L}^{\prime}=\bar{\psi}\left(i \partial_{\mu} \gamma^{\mu}-M+i a \gamma^{0} \gamma^{\mu} \widetilde{F}_{\mu}\right) \psi,
$$

whose equation of motion reads

$$
\left(i \partial_{\mu} \gamma^{\mu}-M+i a \gamma^{0} \gamma^{\mu} \widetilde{F}_{\mu}\right) \psi=0 .
$$

Before studying some properties of such a term, like its connection with the AC effect, we shall give attention to its behavior under special properties, say, gauge invariance, charge conjugation $(C)$, parity $(P)$, and time reversal $(T)$. For this, let us take $\gamma^{0}=\sigma^{z}, \gamma^{1}=i \sigma^{x}$, and $\gamma^{2}=i \sigma^{y}$ as the representation of the Dirac matrices in $2+1$ dimensions.

First, analyzing the behavior of $\mathcal{L}^{\prime}$ under gauge transformations, we may clearly realize its gauge invariance, since

$$
\begin{gathered}
\delta \bar{\psi}=\epsilon \bar{\psi}, \\
\delta \psi=-\epsilon \psi, \\
\delta E_{x}=\epsilon \gamma^{1} \eta, \\
\delta E_{y}=2 \epsilon \gamma^{2} \eta, \\
\delta B=\epsilon \eta,
\end{gathered}
$$

where $\epsilon$ is a global gauge parameter and $\eta$ is a local auxiliary field which helps in the gauge invariance.

On the other hand, using the identity $\gamma^{\mu} \gamma^{\nu}=\eta^{\mu \nu}$ $-i \epsilon^{\mu \nu \kappa} \gamma_{\kappa}$, we may write

$$
i a \bar{\psi} \gamma^{0} \gamma^{\mu} \widetilde{F}_{\mu} \psi=-i a B \bar{\psi} \psi+a \bar{\psi} \vec{\gamma} \cdot \vec{E} \psi
$$

Now, let us see how the terms above behave under $C, P$, and $T$ operations. Let us start off from 


$$
\begin{gathered}
i a B \bar{\psi} \psi \rightarrow-i a B \bar{\psi} \psi, \\
i a B \bar{\psi} \psi \rightarrow-i a B \bar{\psi} \psi, \\
i a B \bar{\psi} \psi \rightarrow+i a B \bar{\psi} \psi ;
\end{gathered}
$$

then, although breaking $C$ and $P$, this term keeps $T$ invariance and so $C P T$ symmetry is preserved. In addition, let us notice that this term provides an extra (imaginary) mass for the fermions when $B \neq 0$ (while its usual counterpart, $f B \bar{\psi} \gamma^{0} \psi$, couples to the electric charge). ${ }^{3}$ lows:

On the other hand, the spatial components behave as fol-

$$
\begin{aligned}
& a \bar{\psi} \vec{\gamma} \cdot \vec{E} \psi \rightarrow+a \bar{\psi} \vec{\gamma} \cdot \vec{E} \psi, \\
& a \bar{\psi} \vec{\gamma} \cdot \vec{E} \psi{ }^{P}++a \bar{\psi} \vec{\gamma} \cdot \vec{E} \psi, \\
& a \bar{\psi} \vec{\gamma} \cdot \vec{E} \psi \rightarrow+a \bar{\psi} \vec{\gamma} \cdot \vec{E} \psi,
\end{aligned}
$$

which tells us that the term above, coupling the current to the electric field, preserves all of these symmetries, and $C P T$ is obviously kept. Here, it should be noted that its usual counterpart, $f \bar{\psi} \gamma^{i} \epsilon^{i j} E^{j} \psi$, which couples the current density to the dual electric field, is $P$ and $T$ odd (while it respects $C P T$, since it is $C$ even). Then, when Lorentz and $C P T$ symmetries are taken into account, we recognize profound differences between the present and the usual nonminimal couplings. Thus, our proposal may be viewed as a low-energy alternative to the standard term, particularly in those cases in which neither $P$ nor $T$ operation is broken.

Hereafter we shall focus our attention on the field produced by the monopole, Eq. (25), and its consequences concerning the AC phase as well. Thus, we shall work with the (Lorentz-violating) Lagrangian below:

$$
\mathcal{L}=\bar{\psi}\left(i \partial_{t} \gamma^{0}-i \partial_{i} \gamma^{i}-M+i a \gamma^{0} \gamma^{i} \widetilde{F}_{i}\right) \psi
$$

which leads to the following equations of motion:

$$
i \partial_{t} \psi(x)=\left[\gamma_{0} \vec{\gamma} \cdot\left(-i \vec{\nabla}+a \vec{E}_{g}\right)+M \gamma_{0}\right] \psi(x)
$$

In addition, it is easy to show that the Lagrangian (52) is invariant under gauge transformations, i.e.,

\footnotetext{
${ }^{3}$ Then, in view of its imaginary nature, we should take it away from Eq. (42) in order to maintain the real character of this Lagrangian. This is done in what follows [see Eq. (52) and related discussion].
}

$$
\begin{gathered}
\delta \bar{\psi}=\epsilon \bar{\psi}, \\
\delta \psi=-\epsilon \psi, \\
\delta E_{x}=\epsilon \gamma^{2} \phi, \\
\delta E_{y}=\epsilon \gamma^{1} \phi,
\end{gathered}
$$

where $\phi$ is a local auxiliary field analog to the $\eta$ field described above.

Now, taking the field generated by a pointlike monopole, $E_{i}=\left(E_{g}\right)_{i}=g \epsilon_{i j} x_{j} / 2 \pi|\vec{x}|^{2}$, and working in the WKB approximation, we have that

$$
\psi(\vec{x}, t)=\psi^{(0)}(\vec{x}, t) \exp \left[-i a \int d l_{i} E_{g}^{i}\right],
$$

with $\psi^{(0)}$ satisfying Eq. (53) when $\vec{E}_{g}$ is vanishing. Now, supposing that the fermion performs a loop, $\alpha_{g}$, around the monopole,

$$
\alpha_{g}=a \oint d l_{i} E_{g}^{i}=\frac{a g}{2 \pi} \oint \epsilon_{i j} d x_{j} \epsilon^{i k} E_{g}^{k}=a g,
$$

which clearly represents the AC phase on the fermion wave function produced by the monopole field $\vec{E}_{g}$. We should stress, once more, that such a phase comes from a $C-, P$-, and $T$-invariant nonminimal coupling.

In this way we have carried the duality symmetry found in the previous section between the electric field produced by a static monopole and the vector potential of a magnetic vortex to the level of quantum mechanics.

Now, in order to study the behavior of the wave functions it is more convenient to work with the second-order differential equation in polar coordinates $(x=r \cos \varphi$ and $y$ $=r \sin \varphi$ ), as follows:

$$
\begin{aligned}
& {\left[\frac{1}{r} \frac{\partial}{\partial r}\left(r \frac{\partial}{\partial r}\right)+\frac{1}{r^{2}}\left(\frac{\partial}{\partial \varphi}+i \alpha\right)^{2}-\alpha s \sigma_{z} \frac{1}{r} \delta(r)+k^{2}\right] \psi(r, \phi)} \\
& \quad=0
\end{aligned}
$$

where $\alpha=a g / 2 \pi, k^{2}=E^{2}-M^{2}$, and we are using $s=+1$ for "spin up" and $s=-1$ for "spin down" (the actual spin of the spinors is $s / 2$ ).

This equation is well known from the Aharonov-Bohm (AB) effect for relativistic particles. In fact, this is a sort of Aharonov-Casher effect since we have a neutral particle in the presence of an electric field, as we have discussed above. Furthermore, the presence of spin leads to the $\delta(r)$ interaction, which mimics the interaction of the spin of the particle with a magnetic vortex (Zeeman effect), and may be interpreted as a contact interaction of the spin with the monopole itself. Although this residual interaction term vanishes outside the location of the monopole, the influence of the monopole on the dynamics of the particles is still felt by the induction of a nontrivial phase, Eqs. (55),(56), and, consequently, affects the phase shift of the scattered wave function. 
In the work of Refs. [20-22] this problem is treated in the context of the $\mathrm{AB}$ effect. The authors adopt different approaches to regularize the delta function potential, which in our case is equivalent to supposing that the radius $R$ of the monopole is finite and is taken to zero at the end of the calculations.

To quote the main results, we consider the upper component of $\psi(r, \varphi)$ and expand it as

$$
\psi_{1}=\sum_{-\infty}^{m=+\infty} f_{m}(r) e^{i m \varphi}
$$

where $f_{m}(r)$ obeys the following equation:

$$
\left[\frac{1}{r} \frac{d}{d r}\left(r \frac{d}{d r}\right)-\frac{(m+\alpha)^{2}}{r^{2}}-\frac{\alpha}{R} \delta(r-R)+k^{2}\right] f_{m}(r)=0
$$

with the boundary conditions

$$
\begin{aligned}
f_{m}(R-\varepsilon) & =f_{m}(R+\varepsilon), \\
\left.R(d / d r) f_{m}\right|_{R-\varepsilon} ^{R+\varepsilon} & =\alpha f_{m}(R),
\end{aligned}
$$

which incorporate the effect of the delta function.

By writing the $f_{m}(r)$ in terms of Bessel functions

$$
f_{m}(r)=\left\{\begin{array}{l}
A_{m} J_{|m+\alpha|}(k r)+B_{m} J_{-|m+\alpha|}(k r), \quad r>R \\
C_{m} J_{m}(k r), \quad r<R
\end{array}\right.
$$

and using Eqs. (60) to determine the coefficients of the Bessel functions, this renormalization method allows for the irregular function $J_{-|m+\alpha|}$ to contribute if the relations

$$
|m|+|m+\alpha|=-\alpha s
$$

and

$$
|m|+\alpha s+1>0
$$

are simultaneously satisfied [20,21].

The same kind of problem was analyzed in [23-25] by using the self-adjoint method and found to be equivalent to the renormalization method if some relations between the self-adjoint parameter and the renormalized coupling constant are satisfied [26].

\section{CONCLUSIONS AND PROSPECTS}

In the present paper, attention was given to Dirac-like monopoles in three-dimensional Abelian Maxwell and Maxwell-Chern-Simons models. Initially, we gave an alternative view of the scalar nature of such objects in a planar world. This was done by carrying out the dimensional reduction of four-dimensional Maxwell theory, enriched by magnetic sources, to three dimensions. There, we realized the appearance of two independent Abelian factors, one related to the usual $A^{\mu}$ potential ( $\vec{E}$ and $\vec{B}$ fields), while the other is implemented by a scalar potential $S$. In addition, we have also verified that the broken Bianchi identity of the $A^{\mu}$ sector (the usual planar electromagnetism), $\partial_{\mu} \widetilde{F}^{\mu}=\chi$, presents a pseudoscalar that is the survivor of the third component of the genuine magnetic four-current.

Furthermore, in analyzing the structure of the solutions of $\partial_{\mu} \widetilde{F}^{\mu}=\chi$, we have realized that it admits a wider class of solutions than so far considered in the literature. Indeed, in Minkowski space-time, we have seen that not only does the azimuthal-like electric field show up, but also magnetic vortexlike solutions may appear as well.

In addition, when neutral matter interacts nonminimally with Dirac-like monopoles in a particular way, say, via $i \gamma^{0} \gamma^{i} \widetilde{F}_{i}$, then an analogue to the Aharonov-Casher effect is exhibited by such particles. We have also found some subtleties which have to be taken into account carefully because of the effective delta function potential whose origin rests on the "contact" interaction between the particle and the monopole. This is still under study, as well as the consequences of the allowed solutions on the angular momentum of the particle and perhaps on the quantization of the parameter $\alpha$.

Before pointing out our prospects, we would like to address once more the issue concerning the Bianchi identity in $2+1$ dimensions. First of all, let us suppose it holds. Now, let us consider a physical system in which magnetic vortices are created, for instance, when the external magnetic field is suitably increased in high- $T_{c}$ superconductor samples. More precisely, let us imagine that one vortex is created at $t=t_{1}$ and at the spatial origin. Then, the superconductor is supplemented by $B_{1}(\vec{x}, t)=b_{1} \delta^{2}(\vec{x}) \Theta\left(t-t_{1}\right)$. On the other hand, since $\partial_{\mu} \widetilde{F}^{\mu}=\partial_{t} B-\nabla \wedge \vec{E}=0$ then $B_{1}$ above must induce a tangential-like electric field $\vec{E}_{1}=b_{1}\left(\hat{e}_{\theta} / r\right) \delta\left(t-t_{1}\right)$, in order to prevent the breaking of the Bianchi identity. Hence, we conclude that the azimuthal-like electric field may appear even in standard planar electromagnetism, say, without Dirac-like objects. Actually, $\vec{E}_{1}$ above survived only at $t$ $=t_{1}$ because we have supposed that the creation of the vortex is also instantaneous. Now, if a finite time is needed for creating such a vortex, then we expect that this electric field will also occur during all this time.

As perspectives for future investigation, we may quote, among others, the issue concerning the effect of the dimensional reduction on the so-called Dirac quantization condition in $3+1$ dimensions and what would be its counterpart in the planar world (as far as we have understood, the Henneaux-Teitelboim condition [12] does not answer for such a point, since it seems to be valid only when the topological mass is nonvanishing).

The relevance of the scalar field $S$ appearing in Sec. II is also under investigation in the context of the so-called statistical field. Actually, by taking an Abelian Lagrangian which contains the usual Maxwell and the $\theta$ term as well, $\theta \widetilde{F}_{\hat{\mu} \hat{\nu}} F^{\hat{\mu} \hat{\nu}}$, in $3+1$ dimensions, we have seen that, after a suitable dimensional reduction scheme, we naturally generate in $2+1$ dimensions a model that encloses the kinetic terms for $A_{\mu}$ and $S$ as well as another one that links both of these fields by means of a Chern-Simons-like term. Indeed, by identifying $a_{\mu}=\partial_{\mu} S$, we clearly realize that such a subsequent model is actually that for the (nondynamical) statis- 
tical field $a_{\mu}$, into which this field enters in order to restore parity symmetry. Further results will appear elsewhere [27].

The discussion raised in the preceding paragraphs may also lead us to interesting results, in particular, for providing a link between fundamental aspects of planar Abelian electrodynamics and condensed matter phenomena, namely, the up-to-date topic of high- $T_{c}$ superconductivity. Still in this line, the study of the interaction between the usual particles and Dirac-like objects may be useful in connection to lowenergy problems.

\section{ACKNOWLEDGMENTS}

E.M.C.A. is financially supported by Fundação de Amparo à Pesquisa do Estado de São Paulo (FAPESP). W.A.M.M. is financially supported by Fundação de Amparo à Pesquisa do Estado de Minas Gerais (FAPEMIG). He also thanks CBPF and GFT/UCP where part of this work was done. This work was also partially supported by Conselho Nacional de Desenvolvimento Científico e Tecnológico (CNPq).
[1] P.A.M. Dirac, Proc. R. Soc. London A133, 60 (1931).

[2] G. 't Hooft, Nucl. Phys. B79, 276 (1974).

[3] A.M. Polyakov, JETP Lett. 20, 194 (1974).

[4] P. Goddard and D. Olive, Rep. Prog. Phys. 41, 1357 (1978).

[5] R. Jackiw and S. Templeton, Phys. Rev. D 23, 2291 (1981); W. Siegel, Nucl. Phys. B156, 135 (1979); J. Schonfeld, ibid. B185, 157 (1981); see also R. Jackiw, in J. A. Swieca Summer School '89, edited by Sociedade Brasileira de Fisica (Sociedade Brasileira de Física, Sao Paulo, 1989); “Topics in Planar Physics."

[6] S. Deser, R. Jackiw, and S. Templeton, Ann. Phys. (N.Y.) 140, 372 (1982); Phys. Rev. Lett. 48, 975 (1982).

[7] F. Wilczek, Phys. Rev. Lett. 48, 1144 (1982); see also A. Lerda, Anyons: Quantum Mechanics of Particles with Fractional Statistics, Lecture Notes in Physics Vol. M14 (Springer, Berlin, 1992).

[8] See F. Wilczek, Fractional Statistics and Anyon Superconductivity (World Scientific, Singapore, 1990).

[9] R. Prange and S. Girvin, The Quantum Hall Effect (Springer, New York, 1987); H. Aoki, Rep. Prog. Phys. 50, 655 (1987); G. Morandi, Quantum Hall Effect (Bibliopolis, Naples, 1988); Z. Zhang, T. Hansson, and S. Kivelson, Phys. Rev. Lett. 62, 980 (1989)

[10] R.B. Laughlin, Science 242, 525 (1988); Phys. Rev. Lett. 60, 2677 (1988); A. Fetter, C. Hanna, and R.B. Laughlin, Phys. Rev. B 39, 9679 (1989); Y.-H. Chen, F. Wilczek, E. Witten, and B. Halperin, Int. J. Mod. Phys. B 3, 1001 (1989); S. Randjbar, A. Salam, and J. Strathdee, ibid. 5, 845 (1991); N. Dorey and N. Mavromatos, Phys. Lett. B 266, 163 (1991).
[11] R. Jackiw, Ann. Phys. (N.Y.) 201, 83 (1990), and related references therein.

[12] M. Henneaux and C. Teitelboim, Phys. Rev. Lett. 56, 689 (1986).

[13] R. Pisarski, Phys. Rev. D 34, 3851 (1986).

[14] W.A. Moura-Melo and J.A. Helayël-Neto, Phys. Rev. D 63, 065013 (2001); W. A. Moura-Melo, Ph.D. thesis, CBPF, 2001; concerning the subject of quantum aspects of planar radiation, see also W.A. Moura-Melo and J.A. Helayël-Neto, Phys. Lett. A 293, 216 (2002).

[15] A. Salam and J. Strathdee, Ann. Phys. (N.Y.) 141, 316 (1982).

[16] J. Stern, Phys. Lett. B 265, 119 (1991); Y. Georgelin and J. Wallet, Mod. Phys. Lett. A 7, 1149 (1992); Phys. Rev. D 50, 6610 (1994); M. Torres, ibid. 46, 2295 (1992); F. Nobre and C. Almeida, Phys. Lett. B 455, 213 (1999).

[17] M. Carrington and G. Kunstatter, Phys. Rev. D 51, 1903 (1995).

[18] Y. Aharonov and D. Bohm, Phys. Rev. 115, 485 (1959).

[19] Y. Aharonov and A. Casher, Phys. Rev. Lett. 53, 319 (1984).

[20] C.R. Hagen, Phys. Rev. Lett. 64, 503 (1990).

[21] C.R. Hagen, Int. J. Mod. Phys. A 6, 3119 (1991).

[22] S. Shunqing and T. Ruibao, Phys. Rev. A 45, 1357 (1992).

[23] M.G. Alford and F. Wilczek, Phys. Rev. Lett. 62, 1071 (1989).

[24] Ph. Gerbert and R. Jackiw, Commun. Math. Phys. 124, 229 (1989).

[25] Ph. Gerbert, Phys. Rev. D 40, 1346 (1989).

[26] R. Jackiw, in M. A. B. Beg Memorial Volume, edited by A. Ali and P. Hoodbhoy (World Scientific, Singapore, 1991).

[27] E. Abreu, J.A. Helayël-Neto, M. Hott, and W.A. Moura-Melo (work in progress). 